David Eisenbud

Nagoya Math. J.

Vol. 54 (1974), 61-67

\title{
ADIC APPROXIMATION OF COMPLEXES, AND MULTIPLICITIES
}

\author{
DAVID EISENBUD
}

\section{1) The Theorem}

In [2, Section 1.6] Peskine and Szpiro prove a theorem on adic approximations of finite free resolutions over local rings which, together with M. Artin's Approximation Theorem [1], allows them to "descend" modules of finite projective dimension over the completions of certain local rings to modules of finite projective dimension over finite étale extensions of those rings. In this note we will prove a more general result, which deals with the change in homology under an adic approximation of any complex of finitely generated modules over a noetherian ring, and which allows one to descend not only modules of finite projective dimension, but also the Euler characteristic or intersection multiplicity of two such modules.

Henceforth, $R$ will be a commutative noetherian ring, and $J$ will be an ideal in the (Jacobson) radical of $R$. If

$$
\underline{C}: \cdots \longrightarrow C_{1} \stackrel{f_{1}}{\longrightarrow} C_{0} \stackrel{f_{0}}{\longrightarrow} C_{-1} \stackrel{f_{-1}}{\longrightarrow} \cdots
$$

is a complex of finitely generated $R$-modules, a $J$-adic approximation of $C$ of order $d=\left(\cdots, d_{1}, d_{0}, d_{-1}, \cdots\right)$ will be a complex

$$
\underline{C}_{\varepsilon}: \cdots C_{1} \stackrel{f_{1}+\varepsilon_{1}}{\longrightarrow} C_{0} \stackrel{f_{0}+\varepsilon_{0}}{\longrightarrow} C_{-1} \stackrel{f_{-1}+\varepsilon_{-1}}{\longrightarrow} \cdots
$$

where

$$
\varepsilon_{i}: C_{i} \longrightarrow J^{d i} C_{i-1}
$$

for all $i$. We will show that if the $d_{i}$ are large enough, then the homology of $\underline{C}$ and the homology of $\underline{C}_{\varepsilon}$ are very much alike.

To be more precise, we note that $\underline{C}$ may be regarded as filtered

Received December 13, 1973. 
complex, where the $p^{\text {th }}$ submodule in the filtration of $C_{n}$ is $J^{p} C_{n}$. We will write $H_{n}(\underline{C})_{p}$ for the $p^{\text {th }}$ submodule in the induced filtration of $H_{n}(\underline{C})$. This filtration on $H_{n}(\underline{C})$ is "J-bonne" in the sense of [3, Section 2A. 5] - that is, $\bigcap_{p \geq 0} H_{n}(\underline{C})_{p}=0$, and for large $p, H_{n}(\underline{C})_{p+1}=J H_{n}(\underline{C})_{p}$. We let $\mathrm{gr} H_{n}(\underline{C})$ be the associated graded module. We will use corresponding notation for the complex $\underline{C}_{\varepsilon}$. We are now ready to state the main result.

THEOREM: Let $R, J, \underline{C}$ be as above. There exists a sequence of positive integers $d=\left(\cdots, d_{1}, d_{0}, d_{-1}, \cdots\right)$ such that if $\underline{C}_{8}$ is a J-adic approximation of $C$ of order $d$, then

1) $\operatorname{gr} H_{n}\left(\underline{C}_{\varepsilon}\right)$ is a subquotient of $\mathrm{gr} H_{n}(\underline{C})$ for all $n$, and

2) if $H_{n}(\underline{C})$ and $H_{n-1}(\underline{C})$ are both annihilated by some power of $J$, then $\operatorname{gr} H_{n}(\underline{C}) \cong \operatorname{gr} H_{n}\left(\underline{C}_{\bullet}\right)$.

It would be interesting to know whether the " $\mathrm{gr}$ " in the conclusion of the theorem could be omitted.

The theorem follows from an analysis of the spectral sequence associated to a filtered complex. As with most such analysis, many variations are possible, in which conclusions slightly weaker than that of 2) are deduced from correspondingly weakened hypotheses.

\section{2) Corollaries}

As above, we suppose that $J$ is an ideal contained in the radical of the noetherian ring $R$. Let $I=\left(g_{1}, \cdots, g_{n}\right)$ be an ideal of $R$ contained in $J$, and let $M$ be a finitely generated $R$-module. Recall that the $I$ depth of $M$ is the length of a maximal $M$-sequence contained in $I$. It is natural to ask what happens to the $I$-depth of $M$ if we replace $I$ by a $J$-adic approximation of $I$; that is, if we replace $I=\Sigma R g_{i}$ by an ideal of the form $I_{\varepsilon}=\Sigma R\left(g_{i}+\varepsilon_{i}\right)$, with $\varepsilon_{i} \in J^{d}$ for large $d$.

COROLLARY 1: With the above notation,

$$
I_{s} \text {-depth } M \geq I \text {-depth } M
$$

if $d$ is sufficiently large.

It is easy to see that the inequality may be strict, even if the $g_{i}$ are a minimal set of generators for $I$.

Proof. Let $\underline{K}$ be the Koszul complex associated to $g_{1}, \cdots, g_{n}, \underline{K}_{s}$ the 
one associated to $g_{1}+\varepsilon_{1}, \cdots, g_{n}+\varepsilon_{n}$. Setting $s=I$-depth $M$, it follows from [3, Section 4A. 2] that $H_{n-s+1}(\underline{K} \otimes M)=0$, and it suffices to show that $H_{n-s+1}\left(\underline{K}_{\varepsilon} \otimes M\right)=0$. But $\underline{K}_{8} \otimes M$ is an approximation of order $(\cdots d, d, d, \cdots)$ of $\underline{K} \otimes M$; so by the theorem, if $d$ is large, $H_{n_{-s+1}}(\underline{K} \otimes M)$ $=0$ as required.

Now suppose that $R$ is local, and that $J$ is its maximal ideal. Let $M$ and $N$ be two $R$-modules such that $M \otimes_{R} N$ has finite length as an $R$-module. Suppose that the projective dimension of $M$ is $t<\infty$. Following [3] we set

$$
\chi(M, N)=\sum_{k \geq 0}(-1)^{k} \text { length }\left(\operatorname{Tor}_{k}(M, N)\right),
$$

the Euler-Poincaré characteristic of $M$ and $N$. Suppose that

$$
\underline{C}: 0 \longrightarrow C_{t} \longrightarrow \cdots \longrightarrow C_{1} \stackrel{f_{1}}{\longrightarrow} C_{0}
$$

and

$$
\underline{C}^{\prime}: \cdots \longrightarrow C_{2}^{\prime} \longrightarrow C_{1}^{\prime} \stackrel{f_{1}^{\prime}}{\longrightarrow} C_{0}^{\prime}
$$

are free resolutions of $M$ and $N$, and that $\underline{C}_{\varepsilon}$ and $\underline{C}_{\varepsilon}^{\prime}$ are approximations to $C$ and $C^{\prime}$ of orders $d=\left(d_{t}, \cdots, d_{1}\right)$ and $d^{\prime}=\left(\cdots, d_{2}, d_{1}\right)$ respectively. Let $M_{s}=$ Coker $f_{1}+\varepsilon_{1}$, and $N_{\varepsilon}=$ Coker $f_{1}^{\prime}+\varepsilon_{1}^{\prime}$, where $\varepsilon_{1}$ and $\varepsilon_{1}^{\prime}$ are the maps appearing in the approximations.

COROLlaRY 2: There exists an integer $d(M, N)$ such that if $d_{j}, d_{j}^{\prime} \geq$ $d(M, N)$ for all $j \leq t$, then

$$
\chi(M, N)=\chi\left(M_{s}, N_{s}\right) .
$$

Of course a similar result can be proved for the partial Euler characteristics, and, using the Koszul complex, for the multiplicity of $N$ with respect to an ideal of definition (see [3] for definitions).

Using this Corollary it follows, precisely as in [2, Corollary 6.3], that questions about Euler characteristics or multiplicities can in some cases be "descended" from the completion of a local ring to a finite étale extension of that ring.

Proof. The modules $\operatorname{Tor}_{k}(M, N)$ can be computed as the homology of the complex $\underline{C} \otimes \underline{C}^{\prime}$, to which the complex $\underline{C}_{\varepsilon} \otimes \underline{C}_{\varepsilon}^{\prime}$ is an approximation. If $d(M, N)$ is chosen large enough to make $\underline{C}_{\varepsilon}$ and the first $t$ steps 
of $\underline{C}_{\text {s }}^{\prime}$ exact, as is possible by the first part of the theorem, then $\operatorname{Tor}_{k}\left(M_{\varepsilon}, N_{\varepsilon}\right)$ will vanish for $k>t$, and will be the homology of $\underline{C}_{\varepsilon} \otimes \underline{C}_{\varepsilon}^{\prime}$ for $k \leq t$. But since $H(\underline{C})=$ Tor $(M, N)$ has finite length, we see from part two of the theorem that if $d(M, N)$ is chosen sufficiently large, length $\left(\operatorname{Tor}_{k}(M, N)\right)=$ length $\left(\operatorname{Tor}_{k}\left(M_{\varepsilon}, N_{\varepsilon}\right)\right.$ ) for every $k$, whence the Corollary.

\section{The Spectral Sequence}

For the proof of the theorem, we will employ the spectral sequence used by Serre in [3]. We will now briefly review the facts (for details, see [3, Section 2A]).

Let

$$
\underline{C}: \cdots \longrightarrow C_{k} \stackrel{f_{k}}{\longrightarrow} C_{k-1} \longrightarrow \cdots
$$

be filtered by subcomplexes:

$$
\underline{C}=\underline{C}_{0} \supseteq \underline{C}_{1} \supseteq \cdots .
$$

The filtration on $\underline{C}$ induces a filtration on $H(\underline{C})$, and the usual spectral sequence

$$
E(\underline{C}) \Rightarrow H(\underline{C})
$$

is defined by

$$
E_{r, p}^{n}(\underline{C})=Z_{r, p}^{n}(\underline{C}) /\left(Z_{r-1, p+1}^{n}(\underline{C})+B_{r-1, p}^{n}(\underline{C})\right)
$$

where

$$
\begin{aligned}
& Z_{r, p}^{n}(\underline{C})=\left\{x \in C_{n, p} \mid f_{n}(x) \in C_{n-1, p+r}\right\} \\
& B_{r, p}^{n}(\underline{C})=\left\{x \in C_{n, p} \mid \exists y \in C_{n+1, p-r} \text { with } f_{n+1}(y)=x\right\}
\end{aligned}
$$

Now consider the situation in which $\underline{C}$ is a complex of finitely generated modules over a noetherian ring $R$, and the filtration on $\underline{C}$ is given by

$$
\underline{C}_{p}=J^{p} \underline{C}
$$

where $J$ is an ideal contained in the Jacobson radical of $R$. In this setup, Serre proves that the above spectral sequence converges, in the sense that for each $n$ there is an integer $r(n)$ such that

$$
\operatorname{gr}\left(H_{n}(\underline{C})\right)=\coprod_{p} E_{r, p}^{n}
$$


for any $r \geq r(n)$. (The numbers $r(n)$ depend on the numbers obtained from the Artin-Rees lemma).

\section{Proof of the Theorem}

Let $R, J, \underline{C}$ be as in the theorem, and let $\underline{C}_{\varepsilon}$ be a $\left(\cdots d_{1}, d_{0}, \cdots\right)$ order approximation to $\underline{C}$. Let $E=E(\underline{C})$ and $F=E\left(\underline{C}_{\varepsilon}\right)$ be the spectral sequences described in the last section, for $\underline{C}$ and $\underline{C}_{8}$. The proof of the theorem depends on the following:

LEMMA : Let $\bar{d}_{n}=\min \left(d_{n+1}, d_{n}\right)$. We have

$$
E_{r, p}^{n}=F_{r, p}^{n}
$$

for all $r \leq \bar{d}_{n}$, and all $p$.

Proof: We must prove that if $r \leq \bar{d}_{n}$, then

$$
Z_{r, p}^{n}(\underline{C})=Z_{r, p}^{n}\left(\underline{C}_{s}\right),
$$

and

$$
Z_{r-1, p+1}^{n}(\underline{C})+B_{r-1, p}^{n}(\underline{C})=Z_{r-1, p+1}^{n}\left(\underline{C}_{\varepsilon}\right)+B_{r-1, p}^{n}\left(\underline{C}_{\varepsilon}\right) .
$$

(Note: we will not prove $B_{r-1, p}^{n}(\underline{C})=B_{r-1, p}^{n}\left(\underline{C}_{\varepsilon}\right)$ !)

To verify the first equality, we simply note that if $x \in C_{n, p}=J^{p} C_{n}$, then

$$
\varepsilon_{n}(x) \in J^{d_{n}} C_{n-1, p}=C_{n-1, p+d_{n}} \subseteq C_{n-1, p+r}
$$

For the second equality it suffices, by the first equality and symmetry, to show that

$$
B_{r-1, p}^{n}(\underline{C}) \subseteq B_{r-1, p}^{n}\left(\underline{C}_{s}\right)+Z_{r-1, p+1}^{n}(\underline{C}) .
$$

To this end, let $x \in B_{r-1, p}^{n}(\underline{C})$, so that there exists $y \in C_{n+1, p-r+1}$ with $f_{n+1}(y)=x$. Of course, we have $\left(f_{n+1}+\varepsilon_{n+1}\right)(y)=x+\varepsilon_{n+1}(y) \in B_{r-1, p}^{n}\left(\underline{C}_{\varepsilon}\right)$. Thus it suffices to show that $\varepsilon_{n+1}(y) \in Z_{r-1, p+1}^{n}(\underline{C})$, or, equivalently, that $f_{n} \varepsilon_{n+1}(y) \in \underline{C}_{n-1, p+r}$. Since $\underline{C}_{\varepsilon}$ is a complex, we have

$$
0=\left(f_{n}+\varepsilon_{n}\right)\left(f_{n+1}+\varepsilon_{n+1}\right)=f_{n} \varepsilon_{n+1}+\varepsilon_{n} f_{n+1}+\varepsilon_{n} \varepsilon_{n+1},
$$

so it is enough to show that $\varepsilon_{n} f_{n+1}(y)=\varepsilon_{n}(x)$ and $\varepsilon_{n} \varepsilon_{n+1}(y)$ are in $C_{n-1, p+r}$; this follows at once, since $r \leq \bar{d}_{n}$.

We now conclude the proof of the theorem. Let $r(n)$ be the "point 
of convergence" of the spectral sequence $E_{r, p}^{n}(\underline{C})_{a} \Rightarrow H_{n}(\underline{C})$, described in the last section.

For part 1) of the theorem, take

$$
d_{n}=\max (r(n), r(n-1))
$$

for all $n$. By the definition of $r(n)$ we will have

$$
\operatorname{gr}\left(H_{n}(\underline{C})\right)=\coprod_{p} E_{r(n), p}^{n} .
$$

By the Lemma, and the choice of $d_{n}$ and $d_{n+1}$, we will have

$$
E_{r(n), p}^{n} \cong F_{r(n), p}^{n},
$$

where $F=E\left(\underline{C}_{\varepsilon}\right)$ is the spectral sequence associated to $\underline{C}_{\varepsilon}$. But

$$
\operatorname{gr} H_{n}\left(\underline{C}_{\varepsilon}\right)=\coprod_{p} F_{\infty, p}^{n}
$$

is a subquotient of

$$
\coprod_{p} F_{r(n), p}^{n}
$$

so part 1) is established.

For part 2), let $p_{0}$ be an integer such that $H_{n}(\underline{C})_{p_{0}}=0=H_{n-1}(\underline{C})_{p_{0}}$; such an integer must exist, since, as we have noted, the filtrations on $H(\underline{C})$ are "J-bonne". We now set

$$
d_{n+1}=d_{n}=d_{n-1}=d=\max \left(r(n), r(n-1), p_{0}\right) .
$$

Suppose that $\underline{C}_{8}$ is a $\left(\cdots d_{n+1}, d_{n}, d_{n-1}, \cdots\right)$-order approximation to $\underline{C}$, and let $F=E\left(\underline{C}_{\varepsilon}\right)$. By the definitions of $r(n), r(n-1)$ and the lemma, we have

$$
\operatorname{gr} H_{n}(\underline{C}) \cong \coprod_{p} E_{d, p}^{n} \cong \coprod_{p} F_{d, p}^{n}
$$

and

$$
\operatorname{gr} H_{n-1}(\underline{C})=\coprod_{p} E_{d, p}^{n-1} \cong \coprod_{p} F_{d, p}^{n-1} .
$$

In particular, $F_{d, p}^{n}=0=F_{d, p}^{n-1}$ if $p \geq p_{0}$, so

$$
F_{r, p}^{n}=0=F_{r, p}^{n-1} \quad \text { if } r \geq d, p \geq p_{0} .
$$

On the other hand, the differential

$$
\partial_{r}: F_{r} \rightarrow F_{r}
$$


of the spectral sequence $F$, has degree $r$, so if $r \geq d$,

$$
\partial_{r}^{n+1}: F_{r}^{n+1} \rightarrow F_{r}^{n}
$$

and

$$
\partial_{r}^{n}: F_{r}^{n} \rightarrow F_{r}^{n-1}
$$

and both zero, by $(* *)$.

Thus $F_{d}=F_{\infty}$, so the proof is completed by $\left(^{*}\right)$.

\section{REFERENCES}

[1] Artin, M.: Algebraic approximation of structures over complete local rings; Publ. Math. I.H.E.S. 36 (1969).

[2] Peskine, C. and Szpiro, L.: Dimension projective finie et cohomologie locale; Publ. Math. I.H.E.S. 42 (1973).

[ 3 ] Serre, J-P.: "Algèbre locale-multiplicités"; Springer Lecture Notes in Math. 11 (1965).

Brandeis University 